\title{
TABLA DE VIDA DE Eurhizococcus colombianus Jakubski (HEMIPTERA: MARGARODIDAE) EN CUATRO ESTRUCTURAS VEGETALES
}

\section{LIFE TABLE OF Eurhizococcus colombianus Jakubski (HEMIPTERA: MARGARODIDAE) ON FOUR PLANT STRUCTURES}

\author{
Helber Arévalo-Maldonadoํㅜ, Martha E. Londoño-Zuluaga², Wilson A. Tobón R. ${ }^{3}$
}

\begin{abstract}
${ }^{1}$ I.A. Docente en formación, Universidad de Ciencias Aplicadas y Ambientales U.D.C.A harevalo@udca.edu.co ${ }^{2}$ I.A. M.Sc. Investigador Master, Corpoica C.I. La Selva Km 7 Vía las palmas, Rionegro Antioquia. mlondono@corpoica.org.co ${ }^{3}$ Tecnologo. Auxiliar Técnico, Corpoica C.I. La Selva Km 7 Vía las palmas, Rionegro Antioquia.
\end{abstract}

Rev. U.D.C.A Act. \& Div. Cient. 15(1): 125 - 133, 2012

RESUMEN

La Perla de tierra Eurhizococcus colombianus Jakubski es una plaga de importancia económica en varios frutales de Colombia, principalmente, la mora. Para establecer cualquier manejo es necesario conocer algunas características biológicas del insecto, como la dinámica poblacional, las relaciones naturales y los factores de mortalidad. Es posible predecir la dinámica poblacional, a través de la cría de insectos y la construcción de tablas de vida, que resumen los datos vitales de una poblacional. El efecto de cuatro estructuras vegetales, tallos y frutos de brevo, bulbos de remolacha y tubérculos de papa criolla, en el ciclo de vida, supervivencia, la tabla de vida y fertilidad fueron determinados. Las ninfas no se desarrollaron en los frutos de brevo y tubérculos de papa criolla por descomposición de las estructuras. En los tallos de brevo y en la remolacha, se logró obtener el desarrollo completo del insecto, encontrando diferencias en la duración del estado de ninfa, la medida longitudinal de los quistes a los 110 días y la supervivencia. Con los datos de la tabla de vida y fertilidad, se determinó que esta especie, en condiciones de laboratorio, presenta un ciclo de vida entre 183-239 días, una baja fecundidad, un poder de multiplicación bajo y un crecimiento poblacional bajo. Los datos indican que los tallos de brevo son adecuados para la cría de E. colombianus y que las medidas de control se deben implementar cuando la plaga se encuentra en las etapas de huevo y ninfa móvil.

Palabras clave: Perla de tierra, técnicas de cría, curva de supervivencia, ciclo de vida, tabla de fertilidad.

\section{SUMMARY}

The ground pearl Eurhizococcus colombianus Jakubski is a pest of economic importance in several Colombian fruittrees, mainly blackberry. For the establishment of any management program it is necessary to know some biological characteristics of this insect such as population dynamics, natural relationships and mortality factors. It is possible to predict the population dynamic through insect rearing and the construction of the life table, which summarizes the data of the population's vitality. The effect of four plant structures, stems and fruits of fig, bulbs of beetroot and tuber of yellow potato, on the life cycle, survival, life table and fertility was determined. The nymphs did not develope on fig fruits and tubers of the yellow potato because of the structures decomposition. On fig stems and beetroot the complete development of the insect's cycle was achieved, finding differences in the duration of the nymphal stage, the longitudinal measure of the cyst at the age of 110 days and the survival. With the life table data and fertility it was determined that this specie under laboratories condition presents a life cycle between 183-239 days, a low fertility, low power of multiplication and low population's growth. The data indicate that fig stems are suitable for rearing $E$. colombianus and that the management measures should be implemented when the pest is at the stage of egg and movable nymph.

Key words: The ground pearl, rearing techniques, survival curve, life cycle, fertility table. 


\section{INTRODUCCIÓN}

Las perlas de tierra son insectos de la familia Margarodidae (Hemiptera: Cocoidea), que se alimentan de la savia elaborada presente en la raíces de plantas hospederas. Este grupo de insectos, se caracteriza por presentar un segundo instar ninfal sésil, llamado quiste y poseer patas protorácicas fuertes, para escavar en estado adulto. Los margaródidos, se alimentan en el primer instar ninfal y de quiste, mientras que en el estado adulto no se alimentan, al estar desprovisto de aparato bucal. La reproducción puede ser sexual o asexual y los instares ninfales pueden ser de tres a cinco en hembras y cinco en machos. La mayoría de especies son univoltinas o, en algunos casos, el ciclo puede durar hasta tres años (Foldi, 2005).

Una especie de la familia Margarodidae de distribución neotropical es Eurhizococcus colombianus (Jakubski, 1965), que se encuentra en varias especies vegetales de importancia económica de Colombia, como la mora (Rubus glaucus), el tomate de árbol (Cyphomandra betacea), el lulo (Solanum quitoense), el manzano (Malus domestica), el brevo (Ficus carica), el aguacate (Persea americana), la feijoa (Feijoa sewolliana), el durazno (Prunus pérsica), la fresa (Fragaria spp.), la curuba (Passiflora spp.) (Castrillón et al. 1998) y la vid (Vitis labrusca) (Kondo \& Gómez, 2008).

De acuerdo con Jakubski, E. colombianus atraviesa por los estados de ninfa, quiste y hembra adulta (Kondo \& Gómez 2008). Similar a Eurhizococcus brasiliensis, las ninfas de $E$. colombianus de primer instar son globosas, de color amarillo pálido y deambulan por el suelo hasta encontrar una raíz para alimentarse; allí, insertan el estilete y se convierten en ninfas de segundo instar, habitualmente conocido como "quiste", al ser apodas con un integumento liso y grueso, formado a partir de secreciones cerosas (Soria \& Dal Conte, 2005; Quiñones et al. 2008). El quiste aumenta de tamaño con el tiempo sugiriendo la presencia de más de un instar ninfal dentro del mismo (Kondo \& Gómez, 2008). Dentro de la caparazón protectora que representa el quiste, se completa el desarrollo ninfal y se forma la hembra, la cual, puede tener reproducción sexual o asexual facultativa; este tipo de reproducción, se presenta en otros margaródidos, como $E$. brasiliensis (Soria \& Dal Conte, 2005). La hembra deposita sus huevos dentro de la protección cerosa en una especie de bolsa, que se forma cuando la hembra retrae el abdomen (Kondo \& Gómez, 2008); todo el conjunto de protección cerosa, la hembra adulta y los huevos, se conoce como ovisaco. En algunos casos, la hembra abandona la protección cerosa y pone los huevos unidos uno tras otro, formando una cadena. La hembra muere cuando realiza la postura, dejando el ovisaco repleto de huevos, que tiene un color blanquecino, con paredes frágiles y quebradizas. Luego, se genera una fase de liberación de huevos y ninfas al romperse las paredes del ovisaco (Soria \& Dal Conte, 2005).

De las plantas mencionadas, la mora es la más afectada por E. colombianus, al reducir la producción hasta en dos toneladas de fruta por hectárea año (Castrillón et al. 2000) y causar la muerte hasta del $100 \%$ de las plantas presentes en un lote (Carvajal \& Guarín, 2002).

Para poder implementar un manejo integrado de las poblaciones de $E$. colombianus es relevante conocer algunas características biológicas, como la duración del ciclo de vida, la mortalidad, la dinámica poblacional y el estado de desarrollo más susceptible e implementar métodos y técnicas apropiadas, con el fin de mantener las poblaciones de plaga por debajo de los niveles capaces de causar daño económico (Kogan, 1998).

Para conocer las características biológicas mencionadas y probar los diferentes métodos de control es necesario tener una metodología de cría, que permita mantener los individuos en condiciones controladas. Las tablas de vida y fertilidad son herramientas prácticas para comparar y desarrollar metodologías de cría y entender los efectos de factores externos, como el empleo de diferentes sustratos alimenticios, diferentes técnicas de cría (Coudron et al. 2002) y diferentes condiciones ambientales (Duarte \& Zenner de Polanía, 2009).

Las investigaciones realizadas para la cría de los margaródidos han seguido las metodologías propuestas por De Klerk (1980) y Hickel et al. (2001), que indican cómo mantener las hembras móviles y los quistes totalmente desarrollados, en suelo húmedo, en condiciones controladas hasta la obtención de huevos y de ninfas neonatas y, luego, criar las ninfas sobre la planta hospedera, en jaulas tipo Galloti de $40 \mathrm{~cm}$ de largo X $50 \mathrm{~cm}$ de alto $X 8 \mathrm{~cm}$ de ancho, en condiciones de casa de malla (Texeira et al. 2002).

A pesar de ser efectivas las metodologías descritas, estas presentan limitantes para la realización de bioensayos, debido a que las plantas hospederas requieren de suelo, que se contamina con entomopatógenos, dificulta la búsqueda de individuos y la mayoría de ninfas no colonizan las raíces (Texeira et al. 2002). Otra limitante está determinada por la planta hospedera, dado que se requiere de plantas homogéneas en el momento del montaje de los experimentos.

Por las razones expuestas y teniendo en cuenta la metodología empleada por Monteiro \& Sauro (1997) para criar a $E$. brasiliensis sobre remolacha, el objetivo del trabajo fue validar la cría de E. colombianus sobre diferentes hospederos, en condiciones controladas, sin emplear suelo, a través de 
los parámetros de la tabla de vida y de fertilidad, el ciclo de vida y las curvas de supervivencia.

\section{MATERIALES Y MÉTODOS}

La investigación, se realizó en cámara climática en el laboratorio de entomología, Centro de Investigación "La Selva", Corporación Colombiana de Investigación Agropecuaria CORPOICA. Las condiciones ambientales de temperatura, humedad relativa y fotofase fueron $20 \pm 5^{\circ} \mathrm{C}, 70 \pm 5 \%$ y 0 horas de luz.

Se recolectaron quistes de $E$. colombianus en avanzado estado de desarrollo, en un lote de mora, en el municipio de Rionegro, Antioquia, situado a N 608'04.8”, O 75²5'10.0”. La cría de los quistes, se realizó de forma individual, en vasos de $3 \mathrm{~mL}$ de capacidad con tapa. Cada vaso, se llenó hasta la mitad con suelo desinfectado por solarización húmeda (camas con suelo-materia orgánica-arena con una relación 4:2:1 humedecidas a capacidad de campo y cubiertas con plástico de invernadero durante 40 días), la humedad del suelo, se mantuvo entre el 60 y $80 \%$ y el quiste se puso sobre el suelo hasta la obtención de los adultos. Mediante observación diaria, se verificaron cambios morfológicos externos en el quiste, visualizando, especialmente, los ovisacos. Los huevos, se mantuvieron en las mismas condiciones hasta la eclosión. Al eclosionar, se tomaron 100 ninfas neonatas procedentes de una hembra para cada tratamiento. Los neonatos, se dispusieron en diez unidades experimentales. La unidad experimental estuvo representada por diez ninfas y una estructura vegetal, la cual, se conservó en espuma floral (Oasis ${ }^{\circledR}$ floral foam), humedecida dentro de una bandeja plástica. Se emplearon cuatro tratamientos, representados en estructuras de cuatro vegetales tallos y frutos de brevo (Ficus carica L.), bulbos de remolacha (Beta vulgaris L.) y tubérculos de papa criolla (Solanum phureja Juzepczuk y Bukasov).

Las hembras adultas que se encontraron sin protección cerosa, se individualizaron en suelo solarizado húmedo, en vasos de $3 \mathrm{~mL}$ de capacidad con tapa inyectada, hasta la obtención de posturas. Cuando se obtuvieron las posturas, los huevos se contabilizaron y se mantuvieron en suelo solarizado, en vasos con las mismas características, hasta la eclosión.

Los parámetros biológicos evaluados fueron: duración de los estados de huevo, ninfa, Hembra móvil, ovisacos (la muerte del adulto se tomó cuando se presentó la ovoposición) y la duración del ciclo de vida (huevo a huevo); la medida longitudinal, desde la parte anterior hasta la parte posterior del quiste, a los 55 y 110 días de desarrollo y de ancho del quiste, en la parte más amplia que se presenta al nivel del abdomen, a los 110 días de desarrollo; la supervivencia, la fertilidad específica, la tasa neta de reproducción (Ro), el intervalo entre generaciones $(\mathrm{T})$, la tasa intrínseca de crecimiento $(\mathrm{rm})$ y la tasa finita de aumento $(\lambda)$. La duración de cada estado y del ciclo de vida total incluyendo la supervivencia; se contabilizó diariamente. Con estos datos, se construyó la curva de supervivencia y de fertilidad específica. Con base en los datos de supervivencia y de ovoposición de cada hembra fue elaborada la curva de fertilidad específica. Posteriormente, se calculó el número de huevos medio por hembra (mx) en cada día de ovoposición (x) considerándose el total de hembras y el índice de supervivencia acumulado de las hembras (lx), durante el período de ovoposición y el número de descendientes que continúan a la siguiente generación (lx.mx). Estos valores constituyen las columnas de la tabla de vida. Con base en la información condensada en la tabla de vida, se estimaron los siguientes parámetros para cada tratamiento, siguiendo a Maia et al. (2000): tasa neta de reproducción (Ro), intervalo entre generaciones $(T)$, tasa intrínseca de crecimiento $\left(r_{m}\right)$ y tasa finita de aumento $(\lambda)$.

Las evaluaciones, se establecieron bajo un diseño experimental completamente al azar. Los datos fueron sometidos a estadística descriptiva y a comparaciones de medias de $\mathrm{t}$ para dos muestras, suponiendo varianzas iguales, con ayuda del programa Microsoft Excel 2007๑.

\section{RESULTADOS Y DISCUSIÓN}

Se logró establecer una metodología de cría bajo condiciones de laboratorio; sin embargo, solo dos estructuras vegetales permitieron el completar el ciclo de vida del insecto: tallos de brevo y bulbos de remolacha. Los frutos de brevo y los tubérculos de papa criolla, se retiraron de la evaluación por descomposición natural.

La duración de la fase de ninfa fue menor (Tabla 1), cuando la perla fue criada en tallos de brevo, posiblemente, a la mayor calidad nutricional del alimento y a una mayor adaptación a esta planta. La adaptación, se pudo evidenciar en campo donde los individuos que se encuentran asociados al brevo presentan un tamaño superior hasta en $1 \mathrm{~mm}$ a los encontrados en otros hospederos. La duración promedio de ninfa, en ambas estructuras vegetales, fue inferior a la encontrada por Castrillón et al. (2000), quienes indicaron una duración de 17 a 20 meses, distribuidos en tres semanas, para el primer instar, y de 16 a 19 meses, en quiste. De la misma forma, los autores presentan una duración de huevo menor y una duración del ciclo mayor a la encontrada en esta investigación. Las diferencias, se dieron a que Castrillón et al. (2000) llevaron la investigación sobre las raíces de otros hospederos: mora, tomate de árbol, lengua de vaca (Rumex crispus) y envidia $\left(R\right.$. acetocella), a una temperatura inferior $\left(15 \pm 3^{\circ} \mathrm{C}\right)$ y emplearon suelo, lo que retarda la colonización de la ninfa sobre la raíz del hospedero. 
Tabla 1. Duración en días de los estados de huevo, ninfa, hembra móvil, del ovisaco y del ciclo de vida de Eurhizococcus colombianus Jakubski.

\begin{tabular}{|l|c|c|c|c|c|}
\hline \multicolumn{1}{|c|}{ Tratamientos } & Huevo* & Ninfa & Hembra móvil & Ovisacos & $\begin{array}{c}\text { Ciclo de vida } \\
\text { huevo-huevo }\end{array}$ \\
\hline Tallo Brevo & $\begin{array}{c}35,03 \pm 9,35 \mathrm{a} \\
(2-51)[101]\end{array}$ & $\begin{array}{c}144,8 \pm 5,98 \mathrm{a} \\
(134-155)[18]\end{array}$ & $\begin{array}{c}53,45 \pm 5,98 \mathrm{a} \\
(4-80)[11]\end{array}$ & $\begin{array}{c}69,78 \pm 20,28 \mathrm{a} \\
(7-80)[14]\end{array}$ & $\begin{array}{c}211,87 \pm 17,72 \mathrm{a} \\
(183-239)[8]\end{array}$ \\
\hline Remolacha & $\begin{array}{c}42 \pm 0 \mathrm{a} \\
(42)[1]\end{array}$ & $\begin{array}{c}149,55 \pm 5,83 \mathrm{~b} \\
(141-155)[9]\end{array}$ & $\begin{array}{c}44,33 \pm 17,61 \mathrm{a} \\
(28-63)[3]\end{array}$ & $\begin{array}{c}77,84 \pm 7,76 \mathrm{a} \\
(52-80)\end{array}$ & $\begin{array}{c}218 \pm 9,89 \mathrm{a} \\
(211-225)[2]\end{array}$ \\
\hline
\end{tabular}

*Los datos presentados solo se pudieron obtener de las hembras móviles.

Medias seguidas por la misma letra no difieren entre sí (prueba de "t") suponiendo varianzas iguales, $\alpha=5 \%$

Los valores entre paréntesis expresan el rango y entre paréntesis el número de unidades experimentales.

La duración del ciclo es cercana a un año, lo que indica que E. colombianus es univoltina y que el recurso alimenticio que explota es pobre en nutrientes (Gullan \& Cranston, 2005).

Los valores de tamaño y de peso son empleados para determinar la nutrición y el crecimiento de los insectos (Klowden, 2007); sin embargo, en este trabajo, solo se tuvo en cuenta el tamaño de quiste de E. colombianus, ya que los quistes no se pueden desprender del sustrato alimenticio. Desprender los quistes del sustrato alimenticio afecta el desarrollo del insecto o, en el peor de los casos, le produce la muerte. La medida longitudinal de los quistes solo presentó diferencias a los 110 días de desarrollo (Tabla 2), las cuales, fueron mayores en las ninfas alimentadas sobre los tallos de brevo. Estas medidas son menores a los quistes colectados en campo, que tienen un tamaño entre 5 y $7 \mathrm{~mm}$; las diferencias se presentaron, por que los tallos presentan un recurso limitado y más difícil de extraer, pero suficiente para que los individuos completen el desarrollo. Una observación encontrada durante la ejecución del presente trabajo es la capacidad que tiene este insecto para completar el ciclo, aun cuando es retirado del sustrato alimenticio; no obstante, hay que tener en cuenta que los individuos retirados en la etapa de quiste no se vuelven a alimentar, presentan un menor tamaño y reducen su progenie. Tal vez estos datos sustentan la separación mecánica de los quistes de la perla de tierra de las raíces de la planta, labor realizada por algunos moricultores del Oriente Antioqueño. Esta labor debe ser evaluada en dos aspectos fundamentales: 1) la relación costo beneficio y, 2) La dispersión de plaga cuando se separa de la planta en los diferentes instares de ninfa.

Tabla 2. Medidas morfométricas de quistes de Eurhizococcus colombianus a los 55 y 110 días de desarrollo de la ninfa.

\begin{tabular}{|c|c|c|c|}
\hline \multirow{2}{*}{ Tratamientos } & \multirow{2}{*}{$\begin{array}{c}55 \text { días } \\
\text { Longitudinal }(\mathrm{mm})\end{array}$} & \multicolumn{2}{|c|}{110 días } \\
\cline { 3 - 4 } & Longitudinal $(\mathrm{mm})$ & Ancho $(\mathrm{mm})$ \\
\hline Tallo Brevo & $\begin{array}{c}1,89 \pm 0,73 \mathrm{a} \\
(0,7-3,28)[62]\end{array}$ & $\begin{array}{c}4,62 \pm 1,15 \mathrm{a} \\
(2,34-6,16)[36]\end{array}$ & $\begin{array}{c}3,21 \pm 0,74 \mathrm{a} \\
(1,92-4,45)[36]\end{array}$ \\
\hline Remolacha & $\begin{array}{c}1,75 \pm 0,47 \mathrm{a} \\
(0,82-3,13)[25]\end{array}$ & $\begin{array}{c}3,94 \pm 1,02 \mathrm{~b} \\
(1,76-5,21)[24]\end{array}$ & $\begin{array}{c}3,04 \pm 0,69 \mathrm{a} \\
(1,99-4,96)[24]\end{array}$ \\
\hline
\end{tabular}

Medias seguidas por la misma letra no difieren entre sí por la prueba de "t" suponiendo varianzas iguales, a 5\% de probabilidad. Los valores entre paréntesis expresan el rango y entre los corchetes el número de unidades experimentales. 
La mayor supervivencia, se obtuvo en las ninfas criadas sobre tallos de brevo (Figura 1). Cuando las ninfas se criaron en tallos de brevo, la curva de supervivencia se ajusta a una curva idealizada tipo II, lo que indica que el porcentaje de individuos que mueren por unidad de tiempo es constante, sin importar el estado de desarrollo, ni el número de supervivientes. Para el caso de las ninfas criadas en remolacha, la curva presenta tres ajustes diferentes durante el ciclo de vida. Desde los cero hasta los 60 días, describe una curva idealizada tipo IV, lo que representa una alta mortalidad de ninfas neonatas; desde los 60 hasta 150 días, una curva tipo II, evidenciándose una mortalidad constante y, finalmente, entre los 150 y 219 días, una curva tipo I, indicando una supervivencia de todos los individuos durante este tiempo y una mortalidad concentrada en estado adulto cuando se presentó la oviposición (Deevey, 1947).

Las supervivencias encontradas no son las ideales para una cría bajo condiciones de laboratorio; sin embargo, conocer la mortalidad de la población en cada etapa de desarrollo permite la evaluación de diferentes agentes de control, diferenciando, de esta manera, la mortalidad causada por factores naturales y la mortalidad producida por el agente de control empleado. En ambos casos, se presentó una alta mortalidad en los primeros días de desarrollo, que se debe a la dificultad que tienen las ninfas para colonizar su hospedero. Observaciones de campo permitieron ver cómo este obstáculo es superado, gracias a que hormigas como Line- pithema sp. cargan las ninfas neonatas hasta las raíces, que proporcionan nutrientes de mejor calidad y en altas concentraciones. Esta asociación es mutualista, donde las hormigas favorecen la dispersión de las perlas de tierra y las protegen de enemigos naturales a cambio de la miel de roció producido por las perlas (Sacchet et al. 2009).

De acuerdo a las curvas de supervivencia encontradas, se recomienda realizar medidas de control en las etapas de desarrollo más susceptibles, debido a que, posiblemente, la supervivencia en campo puede ser cercana al cien por ciento durante todas las etapas de desarrollo, concentrando su mortalidad hasta el momento donde se presenta la ovoposición. La etapa de desarrollo más susceptible, se presenta entre los 0 a 50 días de desarrollo, que incluyen las etapas de huevo y ninfa móvil.

E. colombianus cuando completa su desarrollo ninfal y pasa a la etapa adulta puede salir del quiste y comportarse como hembra móvil o mantenerse dentro de la capa cerosa. Durante la cría, se detectó la salida de algunas hembras del quiste y su posterior regreso al mismo. Cuando los huevos están cercanos a la eclosión, la capa cerosa se torna débil, para permitir la salida de las ninfas. Por esta razón, no se recomienda realizar ninguna acción mecánica cercana a la rizosfera cuando $E$. colombianus se encuentra en esta etapa, ya que esto facilitaría la diseminación de las ninfas en todo el lote.

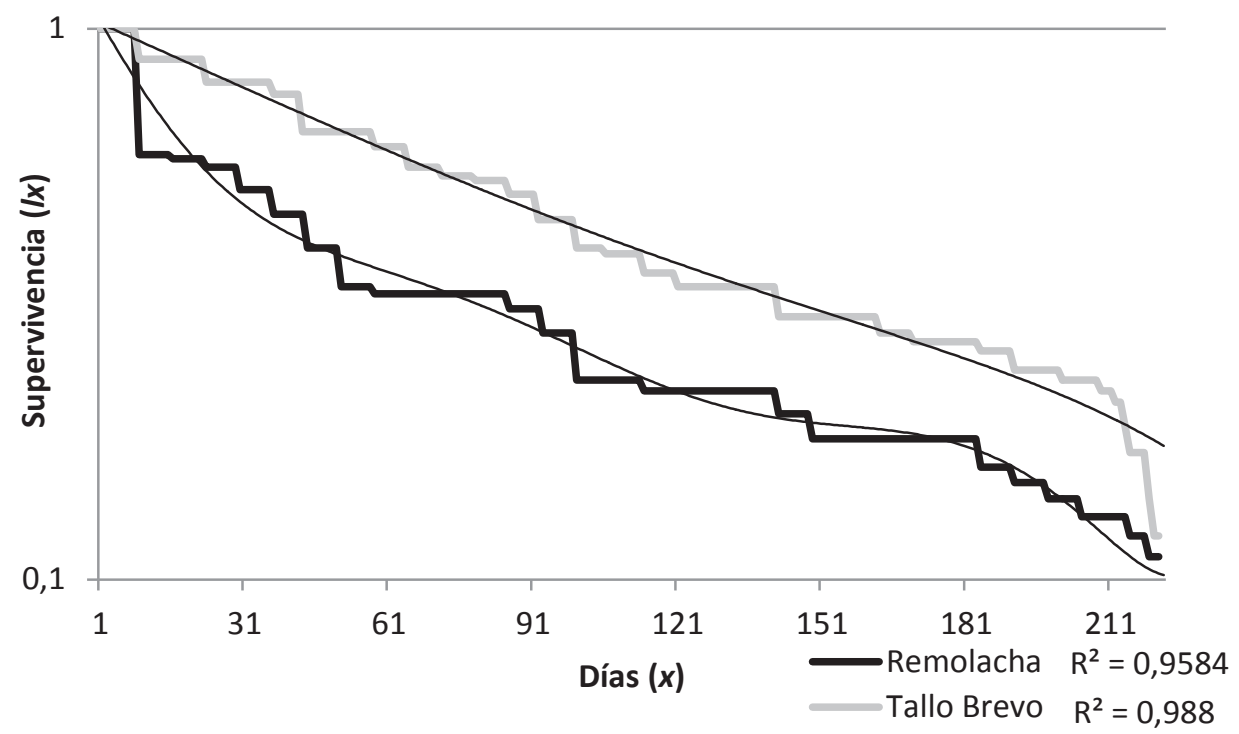

Figura 1. Supervivencia de Eurhizococcus colombianus alimentado en tallos de brevo y bulbos de remolacha. 
La capa cerosa hace resistente al insecto contra aplicaciones de insecticidas de contacto (Botton et al. 2000), por esta razón, es importante conocer el porcentaje de hembras adultas que salen de la protección cerosa y las que se mantienen en ella. Como se observa en la tabla 3, el porcentaje fue afectado notablemente por el alimento consumido durante los instar ninfales; las ninfas alimentadas en remolacha, al llegar al estado adulto, en la mayoría de los casos, se mantuvieron dentro de la capa cerosa (76\%), mientras que las ninfas alimentadas en tallos de brevo presentaron una relación equivalente entre hembras móviles y hembras que permanecen en la capa cerosa.

En la tabla 3 , se consigna la fertilidad y la fecundidad promedio para las hembras móviles. La mayor fecundidad y fertilidad se presentó sobre los tallos de brevo. Los valores encontrados son bajos respecto a los hallados por Castrillón et al. (2000), quienes indicaron una producción de 140 huevos por hembra y a los encontrados en campo de 200 huevos/ hembra en plantas de mora. Nuevamente, se considera que esta diferencia se debe al recurso escaso y, por lo tanto, en una futura metodología, se recomienda buscar la forma para mantener turgente la estructura vegetal y con disponibilidad ilimitada de nutrientes, lo cual, se puede lograr al mantener la estructura en condiciones in vitro o, si es posible, crear una dieta artificial. A pesar de las limitantes mencionadas, la metodología de cría se recomienda, hasta no obtener una mejor propuesta, ya que permite realizar bioensayos, un mayor porcentaje de colonización de las ninfas, realizar un seguimiento diario sin desprender las ninfas de las raíces y emplear sustrato libre de entomopatógenos.

Además en investigaciones posteriores, vale la pena estudiar el efecto de la luz, la humedad, la temperatura, la calidad del alimento sobre la proporción de quistes que dan origen a hembras móviles y a hembras con protección cerosa desde una misma cohorte, para tratar de entender el comportamiento de esta especie, en relación con estos factores.
La tasa neta de reproducción (Ro), intrínseca de crecimiento $(\mathrm{rm})$ y finita de aumento $(\lambda)$ fue superior en los ovisacos, cuyas ninfas fueron alimentadas en los tallos de brevo. En el mejor de los casos, la población presentó un crecimiento del uno por ciento y un individuo es remplazado por 20. Teniendo en cuenta los resultados encontrados y observaciones de campo, se presume que tanto el crecimiento poblacional como la tasa neta de reproducción es superior en campo, lo cual, se debe a que los recursos alimenticios fueron limitados en este experimento.

En cuanto al intervalo entre generaciones $(T)$, no se presentaron diferencias significativas (Tabla 4).

Como se observa en la figura 2, la tasa máxima de aumento de la población alimentada en tallos de brevo, se presentó al día 205, para los ovisacos y al día 209, en las hembras móviles, mientras que la tasa máxima de aumento en la población alimentada en bulbos de remolacha se presentó al día 218, para los ovisacos y al día 201, en las hembras móviles. De estos datos, se puede decir que este parámetro está mediado genéticamente y no nutricionalmente, al presentarse en un periodo de tiempo similar en ambos casos.

De los datos obtenidos del ciclo de vida, de supervivencia y la tabla de vida y de fertilidad, se puede clasificar a E. colombianus como una especie con estrategia ecológica tipo K, al presentar un ciclo de vida largo, una baja fecundidad, un poder de multiplicación bajo y una fluctuación poblacional baja (Pianka, 1970)

Por lo tanto, se recomienda buscar, para el manejo poblacional de esta plaga, agentes de control biológico, que puedan permanecer en la rizosfera por largos periodos de tiempo y la forma de perturbar el hábitat donde se desarrollan.

Como conclusión, se logró establecer una metodología de cría para E. colombianus en condiciones de laboratorio, sin

Tabla 3. Fertilidad, fecundidad, porcentaje de hembras móviles y ovisacos de Eurhizococcus colombianus.

\begin{tabular}{|c|c|c|c|c|}
\hline Tratamientos & $\begin{array}{c}\text { \% Hembras } \\
\text { Móviles }\end{array}$ & \% Ovisacos & $\begin{array}{c}\text { Fecundidad* } \\
\text { (Huevos/hembra) }\end{array}$ & \% de Fertilidad* \\
\hline Estaca Breva & 44 & 56 & 43,37 & 45,64 \\
\hline Remolacha & 23,52 & 76,48 & 18,5 & 5,40 \\
\hline
\end{tabular}

* Los datos presentados corresponden a las hembras móviles. 
Tabla 4. Tasa neta de reproducción (Ro), intervalo entre generaciones $(T)$, tasa intrínseca de crecimiento $\left(\mathrm{r}_{\mathrm{m}}\right)$ y tasa finita de aumento $(\lambda)$ de Eurhizococcus colombianus.

\begin{tabular}{|c|c|c|c|c|}
\hline Tratamientos & Ro & $\mathrm{T}$ & $\mathrm{r}_{\mathrm{m}}$ & $\lambda$ \\
\hline $\begin{array}{c}\text { Tallos brevo Hem- } \\
\text { bras móviles }\end{array}$ & $5,09 \pm 7,85$ & $204,37 \pm 3,85$ & $0,002 \pm 0,01$ & $1,01 \pm 0,01$ \\
\hline $\begin{array}{c}\text { Tallos brevo } \\
\text { Hembras ovisaco }\end{array}$ & $20,07 \pm 10,84$ & $205,59 \pm 4,31$ & $0,013 \pm 0,003$ & $1,01 \pm 0,003$ \\
\hline $\begin{array}{c}\text { Remolacha } \\
\text { Hembras móviles }\end{array}$ & 0,14 & 201 & $-0,009$ & 0,99 \\
\hline $\begin{array}{c}\text { Remolacha } \\
\text { Hembras ovisaco }\end{array}$ & $7,05 \pm 0,79$ & $210,86 \pm 4,84$ & $0,009 \pm 0,0003$ & $1,009 \pm 0,0003$ \\
\hline
\end{tabular}
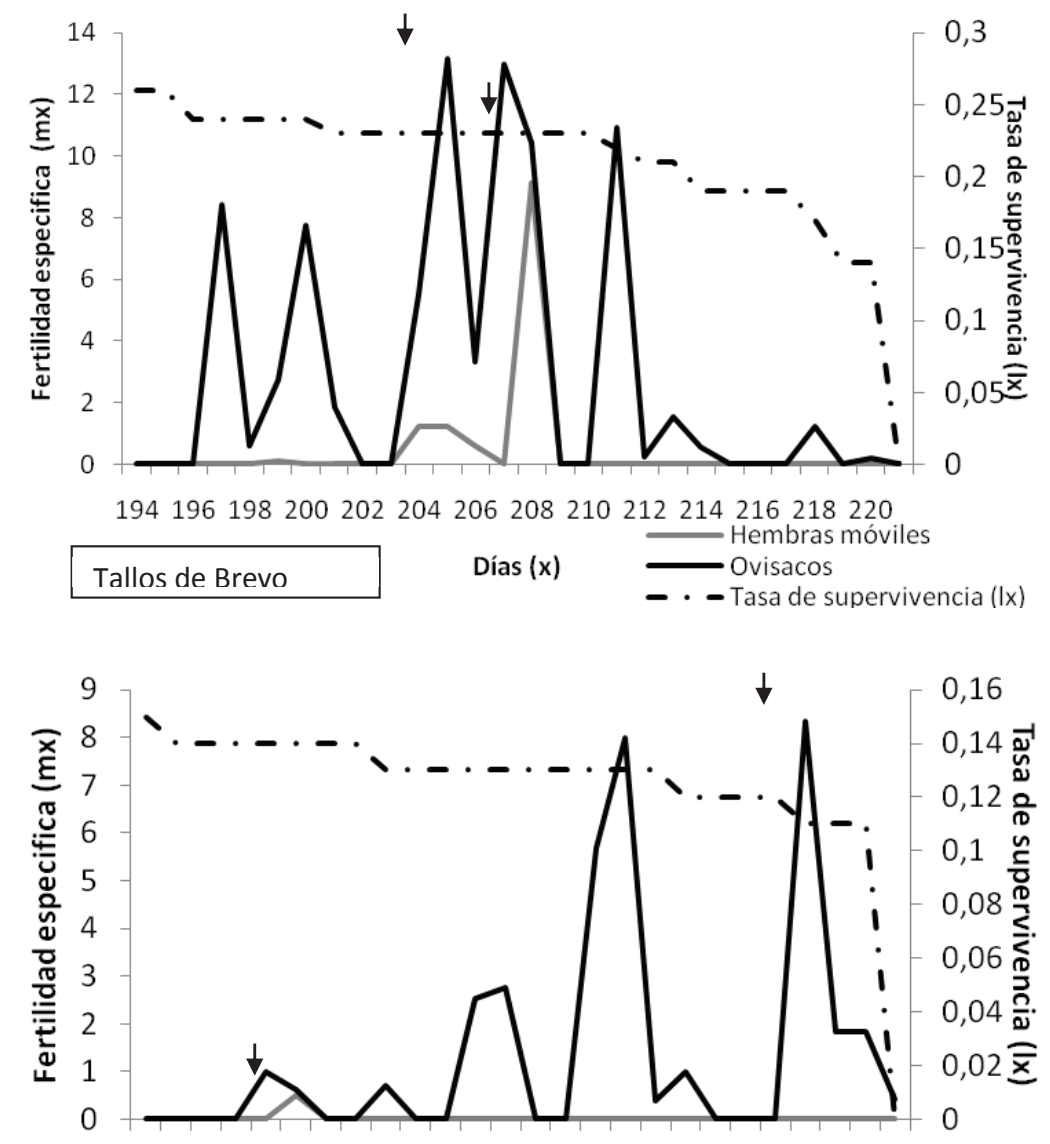

1961982002022042062082102122142162182220

\begin{tabular}{|l|l}
\hline Bulbos de remolacha & Días $(\mathbf{x}) \quad$ Ovisacos \\
\end{tabular}

Figura 2. Tasa de supervivencia (lx) y fertilidad específica (mx) para Eurhizococcus colombianus alimentado en tallos de brevo y bulbos de remolacha. Las flechas indican la tasa máxima de aumento. 
emplear suelo. Las ninfas de perla de tierra, se desarrollaron satisfactoriamente sobre los tallos de brevo y bulbos de remolacha. De acuerdo a los datos obtenidos $E$. colombianus presenta univoltinismo y una estrategia ecológica tipo K. Los resultados obtenidos permiten una relación directa entre el sustrato alimenticio y las diferentes características biológicas evaluadas.

Agradecimientos: Al grupo de entomología del C.I. la Selva de la Corporación Colombiana de Investigación Agropecuaria CORPOICA, al Doctor Saulo de Jesús Soria, por su aportes en el desarrollo del presente trabajo y al Doctor Demian Takumasa Kondo, por la revisión preliminar y aportes. Conflictos de intereses: El manuscrito fue preparado y revisado con la participación de todos los autores, quienes declaramos que no existe ningún conflicto de intereses que ponga en riesgo la validez de los resultados presentados. Financiación: Este estudio fue financiado por CORPOICA, el Ministerio de Agricultura y Desarrollo Rural, la Asociación Hortofrutícola de Colombia ASOHOFRUCOL y la Secretaría de Agricultura y Desarrollo Rural de Antioquia.

\section{BIBLIOGRAFÍA}

1. BOTTON, M.; RODRIGUEZ, E.; SORIA, S.J.; TEXEIRA I. 2000. Bioecología e controle da Pérola-da-terra Eurhizococcus brasiliensis (Hempel 1922) (Hemiptera: Margarodidae) na cultura de videira. EMBRAPA. Circular técnica N²7. p.2-4.

2. CARVAJAL, L.D.; GUARÍN, J.H. 2002. La Perla de tierra Eurhizococcus colombianus Jakubski (Homoptera: Margarodidae) en los frutales de clima frío. En: Centro de Desarrollo Tecnológico de frutales eds. Memorias Cuarto Seminario de Frutales de Clima Frío Moderado. p.153-162.

3. CASTRILLÓN, A.C.; URREA, C.F.; GUEVARA, M.N.; RODRIGUEZ, J.E. 1998. Reacción de diferentes clones de lulo al ataque de la Perla de tierra (Eurhizococcus spp) en zonas de clima frío moderado del departamento de Caldas. En: Centro de desarrollo tecnológico de frutales. eds. Memorias Segundo Seminario de Frutales de Clima Frío Moderado. p.153-160.

4. CASTRILLÓN, A.C.; URREA, C.F.; GUEVARA, M.N.; PINEDA, S.M. 2000. Algunos aspectos biomorfológicos y agroecológicos de la Perla de tierra en zonas Fn. En: Centro de desarrollo tecnológico de frutales eds. Memorias Tercer Seminario de Frutales de Clima Frío Moderado. p.125-131.
5. COUDRON, T.A.; WITTMEYER, J.; KIM, Y. 2002. Life history and cost analysis for continuous rearing of Podisus maculiventris (Say) (Heteroptera: Pentatomidae) on a zoophytophagous artificial diet. J. Econ. Entomol. 95(6):1159-1168.

6. DE KLERK, C.A. 1980. Biology of Margarodes uredendalensis De Klerk (Coccoidea: Margarodidae). South Afr. J. Enol. Vitic. 1:45-58. Disponible desde Internet en http://www.sasev.org/journal-sajev/sajev-articles/ volume-1-1/art6\%20biology\%20of\%20margarodes\%20vredendalensis.pdf [con acceso 24/09/2011].

7. DEEVEY, E.S. 1947. Life table for natural populations of animals. Quart. Rev. Biol. 22:283-314.

8. DUARTE, H.W.; ZENNER DE POLANÍA, I. 2009. Efecto de la temperatura sobre el desarrollo de Eriopis connexa connexa (Germar) (Coleoptera: Coccinellidae). Rev. U.D.C.A Act. \& Div. Cient. 12(2):135-145.

9. FOLDI, I. 2005. Ground pearls: a generic revision of the Margarodidae sensu stricto (Hemiptera: Sternorrhyncha: Coccoidea). Ann. Soc. Entomol. France. 41(1):81-125.

10. GULLAN, P.J.; CRANSTON, P.S. 2005. An outline of entomology. Ed. Blackwell Ltda. (USA). 511p.

11. HICKEL, E.R.; PERUZZO, E.L.; SCHUCK, E. 2001. Controle da pérola-da-terra, Eurhizococcus brasiliensis (Hempel) (Homoptera: Margarodidae), através da insetigação. Neotrop. Entomol. 30(1):127132.

12. KLOWDEN, M.J. 2007. Physiological systems in insects. Ed. Elsevier Inc. (USA). 688p.

13. KOGAN, M. 1998. Integrated pest management: historical perspectives and contemporary developments. Ann. Rev. Entomol. 43:243-270.

14. KONDO, D.T.; GÓMEZ, C.E. 2008. La Perla de tierra, Eurhizococcus colombianus Jakubski. Una nueva plaga de la Vid, Vitis labrusca L. en el Valle del Cauca, Colombia. Corporación Colombiana de Investigación Agropecuaria CORPOICA. Nov. Técn. 9(10):34-40.

15. MAIA, A.; LUIZ, A.; CAMPANHOLA, C. 2000. Statistical inference on associated fertility life table parameters using Jackknife technique: computational aspects. J. Econ. Entom. 93(2):511-518. 
16. MONTEIRO, L.B.; SAURO, R. 1997. Curva de crescimento e criação de Eurhyzococcus brasiliensis (Hempel) (Homoptera: Margarodidae) sobre beterraba em laboratório. Anais da Soc. Entomol. Brasil, Piracicaba. 25(3):563-565.

17. PIANKA, E.R. 1970. On r- and K-selection. Am. Natur. 104(940):592-597.

18. QUIÑONES, W.; VICENTE, B.; TORRES, F.; ARCHBOLD, R.; MURILLO, W.; LONDOÑO, M.; ECHEVERRI, F. 2008. Chemical composition of ground pearl (Eurhizococcus colombianus) Cyst. Molecules. 13(1):190-194. Disponible desde Internet en http://www.mdpi.org/molecules (con acceso el 16/08/2008).

19. SORIA, S. DE J.; DAL CONTE, A.F. 2005. Bioecologia e controle das pragas da videira. Embrapa. Circu- lar Técnica 63. Bento Goncalves, RS Dezembro. 20 P. ISSN 1808-6810. Disponible desde Internet en http:/www.cnpuv.embrapa.br/publica/circular/ cir063.pdf (con acceso 16/08/2008).

20. SACCHET, F.; BOTTON, M.; DIEHL, E. 2009. Ant species associated with the dispersal of Eurhizococcus brasiliensis (Hempel in Wile) (Hemiptera: Margarodidae) in vineyards of Serra Gaucha, Rio Grande do Sul. Sociobiology. 54:943-954.

21. TEXEIRA, I.; BOTTON, M.; ENIMAR, L.A. 2002. Avaliação de Inseticidas Visando ao Controle de Eurhizococcus brasiliensis (Hempel) (Hemiptera: Margarodidae) em Novos Plantios de Videira. Neotrop. Entom.. 31(3):457-461.

Recibido: Enero 26 de 2012

Aceptado: Mayo 3 de 2012 